\title{
Generalized Space-Time Shift Keying Designed for Flexible Diversity-, Multiplexing- and Complexity-Tradeoffs
}

\author{
Shinya Sugiura, Member, IEEE, Sheng Chen, Fellow, IEEE, and Lajos Hanzo, Fellow, IEEE
}

\begin{abstract}
In this paper, motivated by the recent concept of Spatial Modulation (SM), we propose a novel Generalized Space-Time Shift Keying (G-STSK) architecture, which acts as a unified Multiple-Input Multiple-Output (MIMO) framework. More specifically, our G-STSK scheme is based on the rationale that $P$ out of $Q$ dispersion matrices are selected and linearly combined in conjunction with the classic PSK/QAM modulation, where activating $P$ out of $Q$ dispersion matrices provides an implicit means of conveying information bits in addition to the classic modem. Due to its substantial flexibility, our G-STSK framework includes diverse MIMO arrangements, such as SM, Space-Shift Keying (SSK), Linear Dispersion Codes (LDCs), Space-Time Block Codes (STBCs) and Bell Lab's Layered SpaceTime (BLAST) scheme. Hence it has the potential of subsuming all of them, when flexibly adapting a set of system parameters. Moreover, we also derive the Discrete-input Continuous-output Memoryless Channel (DCMC) capacity for our G-STSK scheme, which serves as the unified capacity limit, hence quantifying the capacity of the class of MIMO arrangements. Furthermore, EXtrinsic Information Transfer (EXIT) chart analysis is used for designing our G-STSK scheme and for characterizing its iterative decoding convergence.
\end{abstract}

Index Terms-Diversity and multiplexing tradeoff, space-time shift keying, spatial modulation, linear dispersion code, maximum likelihood detection, multiple antenna array.

\section{INTRODUCTION}

$\mathbf{O}$ VER the last two decades diverse Multiple-Input Multiple-Output (MIMO) arrangements have been developed for achieving diversity, multiplexing and/or beamforming gains [1]. For example, while Bell Lab's Layered Space-Time (BLAST) scheme [2] was designed for high-rate transmission, the class of Space-Time Block Codes (STBCs) [3] was developed for achieving a beneficial diversity gain. Furthermore, in [4] Hassibi and Hochwald proposed the unified space-time concept of Linear Dispersion Codes (LDCs), which strikes a flexible tradeoff between the attainable diversity and multiplexing gains.

Manuscript received January 14, 2010; revised July 29, 2010 and October 26, 2010; accepted December 25, 2010. The associate editor coordinating the review of this paper and approving it for publication was K. B. Lee.

S. Sugiura was with the School of Electronics and Computer Science, University of Southampton, Southampton, SO17 1BJ, UK, and is currently with the Toyota Central Research and Development Laboratories, Inc., Aichi, 480-1192, Japan (e-mail: sugiura@mosk.tytlabs.co.jp).

S. Chen and L. Hanzo are with the School of Electronics and Computer Science, University of Southampton, Southampton, SO17 1BJ, UK (e-mail: $\{1 \mathrm{~h}$, sqc $\} @$ ecs.soton.ac.uk).

The financial support of the EU under the auspices of the Optimix project and of the EPSRC UK is gratefully acknowledged.

Digital Object Identifier 10.1109/TWC.2011.012411.100065

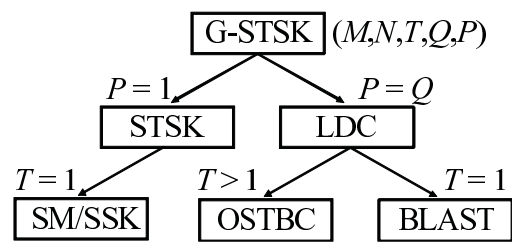

Fig. 1. Relationship between our G-STSK scheme and other MIMO schemes.

Recently, Mesleh et al. proposed the sophisticated concept of Spatial Modulation (SM) [5], [6] $]^{1}$, which employs a novel MIMO encoding principle, where the transmitter activates one out of $M$ transmit Antenna Elements (AEs), whose antennaactivation process acts as an additional means of conveying information bits, and then only the activated antenna transmits a signal modulated with the aid of the classic $\mathcal{L}$-point constellation, such as Phase-Shift Keying (PSK) and Quadrature Amplitude Modulation (QAM). Unlike BLAST, the SM does not transmit simultaneously via $M$ AEs, hence single-antennabased low-complexity ML detection can be employed at the receiver, while dispensing with symbol-level Inter-Antenna Synchronization (IAS) at the transmitter. The special case of SM is constituted by the scenario, where we deactivate the classic PSK/QAM signaling and simply use the presence or absence of energy assigned to a specific antenna, which is also referred to as Space Shift Keying (SSK) [8]. The SSK was investigated both for uncoded and for turbo-coded scenarios, while the optimal ML detector designed for the uncoded SM/SSK scheme was presented in [9]. Although SM/SSK has the potential of outperforming other MIMO arrangements [5]-[9], SM/SSK was not designed for achieving any transmit diversity gain and hence has to rely on the provision of receive diversity in order to combat the effects of fading channels.

Against this background, the main contribution of this paper is that we propose a Generalized Space-Time Shift Keying ( $G$ STSK) scheme, where the SM/SSK concept is extended to include two dimensions, namely space as well as time, and hence it becomes capable of striking a flexible tradeoff between the attainable diversity and multiplexing gains. More specifically, in G-STSK $P$ out of $Q$ dispersion matrices are activated during each transmission interval. As shown in Fig. 1, owing to its high flexibility, the G-STSK framework subsumes most of the above-mentioned MIMO arrangements, such as SM/SSK, LDC, STBC as well as BLAST, and therefore has the potential of flexibly mimicking all of them. Additionally, we conceive the

\footnotetext{
${ }^{1}$ The original concept of SM dates back to [7], although in this scheme the transmitter has to simultaneously activate multiple antenna elements, which is different from [5], [6].
} 


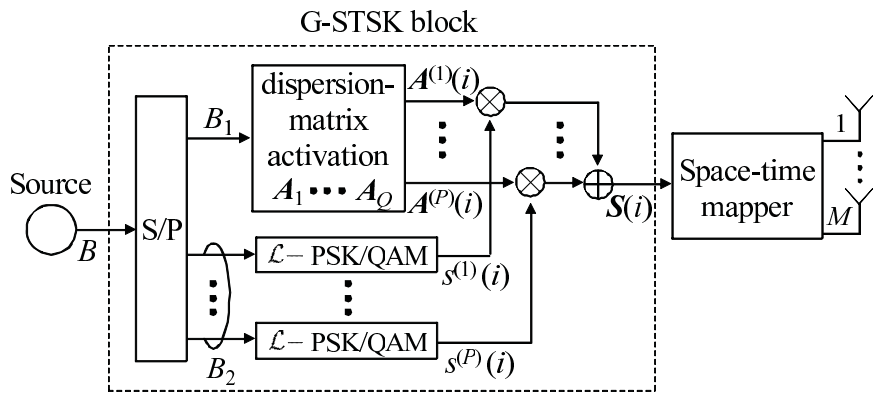

Fig. 2. Transmitter structure of our G-STSK scheme.

optimal ML detector designed for uncoded G-STSK systems and the soft-demodulator conceived for the coded G-STSK systems. Moreover, we also derive the Discrete-input Continuousoutput Memoryless Channel (DCMC) capacity [10] of our $G$-STSK scheme, which serves as the unified capacity, hence characterizing the class of MIMO arrangements, including the ones shown in Fig. 1. EXtrinsic Information Transfer (EXIT) chart analysis [11] is invoked for designing our G-STSK scheme and for characterizing its iterative detection process.

The remainder of this paper is organized as follows. Section II introduces the system model of our G-STSK scheme, and then both the corresponding hard- and soft-decision detectors are presented in Section III. In Section IV we derive the DCMC capacity of our G-STSK scheme, while Section V provides our performance results. Finally, the paper is concluded in Section VI.

\section{System Model}

In this section we introduce our G-STSK scheme and then demonstrate that it subsumes many MIMO schemes as its special cases.

Let us first consider the general block-based space-time system model of [1]

$$
\boldsymbol{Y}(i)=\boldsymbol{H}(i) \boldsymbol{S}(i)+\boldsymbol{V}(i),
$$

where $\boldsymbol{Y}(i) \in \mathcal{C}^{N \times T}$ are the signals received at the receiver equipped with $N$ AEs, while $\boldsymbol{S}(i) \in \mathcal{C}^{M \times T}$ represents the space-time codewords transmitted over $T$ symbol durations from the transmitter having $M$ AEs. Furthermore, transmission $i$ represents the block index. It is also assumed that each component of the channel matrix $\boldsymbol{H}(i) \in \mathcal{C}^{N \times M}$ and the noise matrix $\boldsymbol{V}(i) \in \mathcal{C}^{N \times T}$ obeys the complex-valued Gaussian distribution of $\mathcal{C N}(0,1)$ and $\mathcal{C N}\left(0, N_{0}\right)$, respectively, where $N_{0}$ represents the noise variance. Here, we note that the channel model employed corresponds to a Rayleigh fading scenario.

\section{A. G-STSK Modulation}

Fig. 2 shows the schematic of our G-STSK's transmitter. Let us assume that $Q$ dispersion matrices $\boldsymbol{A}_{q^{\prime}} \in \mathcal{C}^{M \times T}\left(q^{\prime}=\right.$ $1, \cdots, Q)$ are assigned to the transmitter in advance of transmissions. Furthermore, let us introduce the parameter $P$ for representing the number of dispersion matrices, which are activated during each space-time block interval $T$. In the G-STSK bit-to-symbol mapping scheme, $B=\log _{2} f(Q, P)+P \log _{2} \mathcal{L}$ bits per block are mapped to a space-time codeword $\boldsymbol{S}(i)$, where $f(Q, P)$ is calculated from $Q$ and $P$ as $f(Q, P)=2^{\iota}$, while the integer $\iota$ satisfies the following inequality ${ }^{2}$

$$
2^{\iota} \leq\left(\begin{array}{c}
Q \\
P
\end{array}\right)<2^{\iota+1}
$$

Firstly, $B$ input bits are S/P converted to $B_{1}=\log _{2} f(Q, P)$ bits and $B_{2}=P \log _{2} \mathcal{L}$ bits. Then, at the dispersion-matrix activation block of Fig. 2, $P$ out of $Q$ pre-assigned dispersion matrices $\boldsymbol{A}_{q^{\prime}} \in \mathcal{C}^{M \times T}\left(q^{\prime}=1, \cdots, Q\right)$ are activated according to $B_{1}$ input bits, in order to have $\boldsymbol{A}^{(p)}(i)(p=1, \cdots, P)$. By contrast, according to $B_{2}$ input bits, $P$ number of $\log _{2} \mathcal{L}$ bits are separately modulated by the classic $\mathcal{L}$-point PSK/QAM modulation scheme, giving rise to the symbols $s^{(p)}(i)(p=$ $1, \cdots, P)$. Finally, the space-time codeword $\boldsymbol{S}(i)$ is generated as follows:

$$
\boldsymbol{S}(i)=\sum_{p=1}^{P} s^{(p)}(i) \boldsymbol{A}^{(p)}(i),
$$

where we have the power constraint of

$$
\operatorname{tr}\left[\boldsymbol{A}_{q^{\prime}} \boldsymbol{A}_{q^{\prime}}^{H}\right]=\frac{T}{P}\left(q^{\prime}=1, \cdots, Q\right),
$$

in order to maintain a unity total transmission power per symbol. Here, $\operatorname{tr}[\cdot]$ represents the trace operation. We note that as visualized in Fig. 3, each of the P PSK/QAM symbols $s^{(p)}(i)$ is dispersed both to the $M$ spatial and $T$ time dimensions, with the aid of the activated dispersion matrices $\boldsymbol{A}^{(p)}(i)$.

Hence, our G-STSK scheme has a set of parameters given by $M, N, T, Q$ and $P$. Therefore we employ the parameterbased system description of G-STSK $(M, N, T, Q, P)$ for simplicity. Additionally, the normalized throughput $R$ of our GSTSK scheme is given by

$$
R=\frac{B}{T}=\frac{\log _{2} f(Q, P)+P \log _{2} \mathcal{L}}{T} \text { (bits/symbol). }
$$

To be more specific, in Table I we exemplify the bitto-symbol mapping rule of Quadrature Phase-Shift Keying (QPSK)-modulated $(\mathcal{L}=2) \mathrm{G}-\operatorname{STSK}(M, N, T, 4,2)$, where we have $f(Q, P)=2^{\iota}=4$, according to Eq. (2). As seen in Table $\mathrm{I}$, the $B=4$ input bits are $\mathrm{S} / \mathrm{P}$ converted to $B_{1}=2$ bits and $B_{2}=2$ bits. According to the $B_{1}$ bits, $P=2$ out of $Q=4$ dispersion matrices are selected as $\boldsymbol{A}^{(1)}(i), \boldsymbol{A}^{(2)}(i)$, while the $B_{2}$ bits generate the $P=2$ BPSK symbols $s^{(1)}(i), s^{(2)}(i)$. Finally, the space-time codeword $\boldsymbol{S}(i)$ is generated as $\boldsymbol{S}(i)=s^{(1)}(i) \boldsymbol{A}^{(1)}(i)+s^{(2)}(i) \boldsymbol{A}^{(2)}(i)$.

To elaborate a little further, according to [12], the maximum achievable diversity order $D$ of our G-STSK scheme is given by

$$
D=N \cdot \min (M, T),
$$

${ }^{2}$ Although $f(Q, P)$ represents the $P$-out-of- $Q$ dispersion-matrix activation process, which has a maximum of $\left(\begin{array}{c}Q \\ P\end{array}\right)$ possibilities, the relationship of Eq. (2) restricts $\log _{2} f(Q, P)$ to be an integer number for the sake of facilitating a simple natural bit-to symbol mapping. For example, assume that we have $Q=5$ dispersion matrices and activate $P=2$ out of them during each signaling block interval at the G-STSK transmitter. In this case, the potential combinations for activating $P=2$ out of $Q=5$ dispersion matrices is $\left(\begin{array}{c}Q \\ P\end{array}\right)=\left(\begin{array}{l}5 \\ 2\end{array}\right)=10$. However, we only use $2^{\iota}=2^{3}=8$ out of the 10 combinations. 
This article has been accepted for inclusion in a future issue of this journal. Content is final as presented, with the exception of pagination. SUGIURA et al: GENERALIZED SPACE-TIME SHIFT KEYING DESIGNED FOR FLEXIBLE DIVERSITY-, MULTIPLEXING- AND COMPLEXITY-TRADEOFFS 3

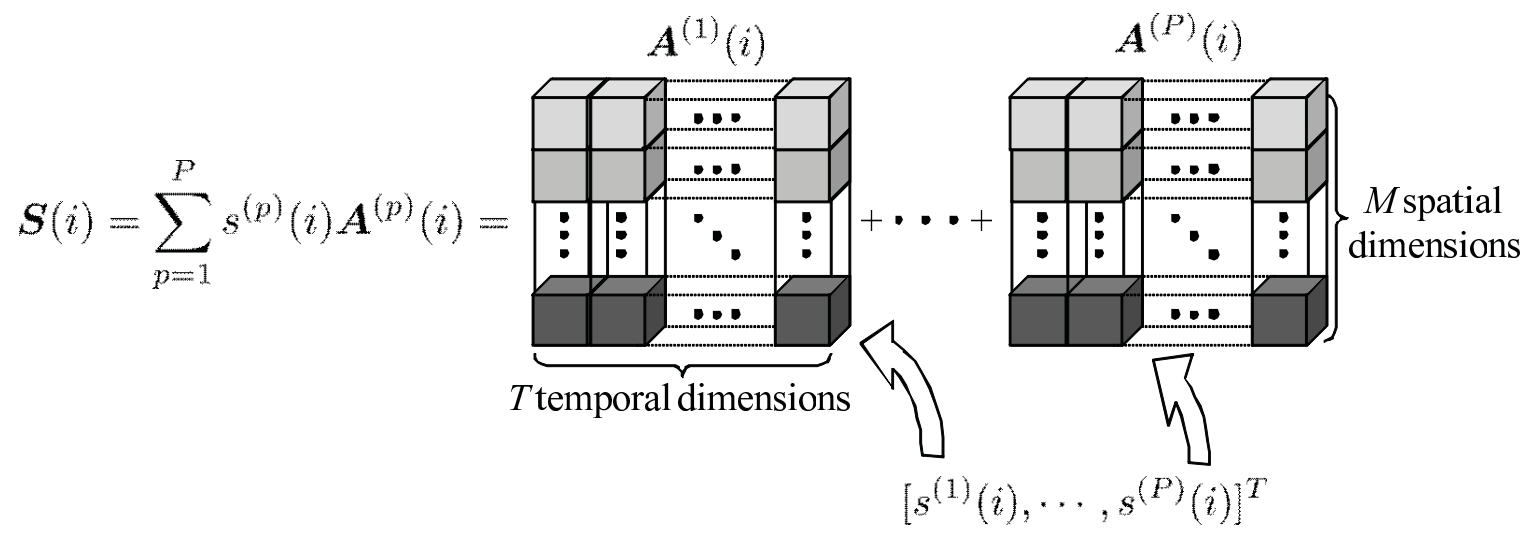

Fig. 3. The space-time codeword $\boldsymbol{S}(i)$ formulated based on Eq. (3).

TABLE I

Example of G-StSK $(M, N, T, 4,2)$ Modulation Scheme, Mapping $B=4$ Bits Per Space-Time Block, with the Aid of BPSK CONSTELLATION

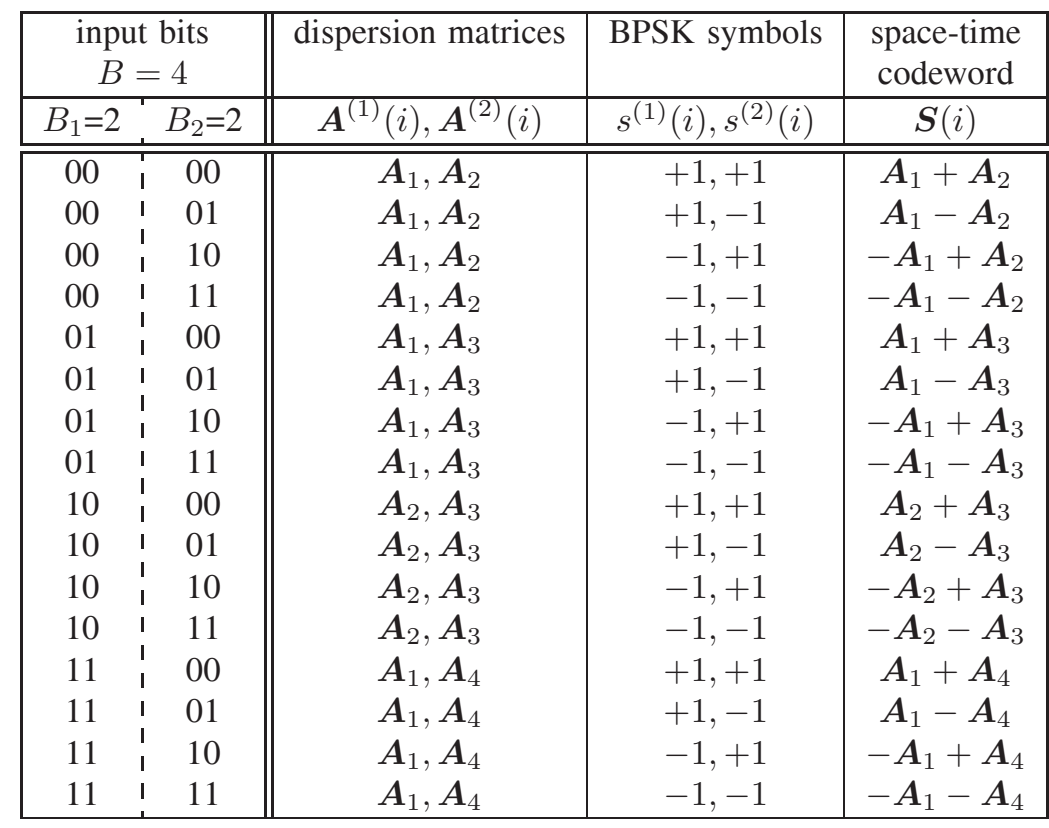

where $N$ and $\min (M, T)$ indicate the attainable receive and the transmit diversity gains, respectively. This indicates that the reduction in $T$ may give rise to the reduction of computational complexity as well as to the enhancement of the normalized throughput in Eq. (5) at the cost of a reduced diversity gain.

\section{B. Relationship Between Our G-STSK Scheme and Conven- tional MIMO Arrangements}

Next, we will demonstrate that our G-STSK scheme includes the class of MIMO arrangements shown in Fig. 1.

1) SM/SSK: The conventional SM/SSK schemes [5], [6], [8], [9] may be derived by the $\operatorname{G-STSK}(M, N, 1, Q=M, 1)$ scheme employing the dispersion matrices of

$$
\boldsymbol{A}_{1}=\left[\begin{array}{c}
1 \\
0 \\
\vdots \\
0
\end{array}\right], \boldsymbol{A}_{2}=\left[\begin{array}{c}
0 \\
1 \\
\vdots \\
0
\end{array}\right], \cdots, \boldsymbol{A}_{Q}=\left[\begin{array}{c}
0 \\
0 \\
\vdots \\
1
\end{array}\right],
$$

where the number of dispersion matrices $Q$ is set to the number of the transmit antennas $M$. As seen in Eq. (6), SM/SSK was not designed for exploiting any transmit diversity, due to the constraint of $T=1$. To be specific, the encoded space-time codeword $\boldsymbol{S}(i)$ may be represented as

$$
\begin{aligned}
\boldsymbol{S}(i) & =\left[\begin{array}{c}
0 \\
\vdots \\
0 \\
s^{(1)}(i) \\
0 \\
\vdots \\
0
\end{array}\right] \leftarrow q \text { th element } \\
& \left.=0 \cdot \boldsymbol{A}_{1}+\cdots+0 \cdot \boldsymbol{A}_{q-1} \leftarrow \text { (deactivated matrices }\right) \\
& +s^{(1)}(i) \cdot \boldsymbol{A}_{q} \leftarrow(\text { activated matrix }) \\
& \left.+0 \cdot \boldsymbol{A}_{q+1}+\cdots+0 \cdot \boldsymbol{A}_{Q}, \leftarrow \text { (deactivated matrices }\right)
\end{aligned}
$$


where we assume that the $q$ th $\mathrm{AE}$ is activated in the $i$ th block duration.

2) $L D C$ : According to the system model of [12], our GSTSK framework associated with $P=Q$ has an identical system model to that of LDCs, where all of the $Q$ preassigned dispersion matrices are used for the linear space-time dispersion of classic PSK/QAM symbols. We note here that as implied by the relation of $B_{1}=\log _{2} f(Q, P)=0$ in Eq. (2), no additional information is transmitted with the aid of the dispersion-matrix activation process in the LDC arrangement. In LDCs, the space-time codeword $\boldsymbol{S}(i)$ may be written as

$$
\boldsymbol{S}(i)=s^{(1)}(i) \cdot \boldsymbol{A}_{1}+\cdots+s^{(Q)}(i) \cdot \boldsymbol{A}_{Q} .
$$

3) STBC: A class of Orthogonal STBCs (OSTBCs) is also subsumed by G-STSK upon setting $P=Q$ and using appropriately designed dispersion matrices, depending on the space-time codewords employed. For example, consider an $(M \times N)=(2 \times 2)$ QPSK-modulated Alamouti STBC [13]. Then the space-time codeword $\boldsymbol{S}(i)$ of Eq. (1) may be expressed as

$$
\begin{aligned}
& \boldsymbol{S}(i)=\frac{1}{\sqrt{2}}\left[\begin{array}{cc}
s_{1} & s_{2} \\
-s_{2}^{*} & s_{1}^{*}
\end{array}\right] \\
& =\underbrace{\left[\begin{array}{cc}
\frac{1}{2} & 0 \\
0 & \frac{1}{2}
\end{array}\right]}_{\boldsymbol{A}_{1}} \sqrt{2} \alpha_{1}+\underbrace{j\left[\begin{array}{cc}
\frac{1}{2} & 0 \\
0 & -\frac{1}{2}
\end{array}\right]}_{\boldsymbol{A}_{2}} \sqrt{2} \beta_{1} \\
& +\underbrace{\left[\begin{array}{cc}
0 & \frac{1}{2} \\
-\frac{1}{2} & 0
\end{array}\right]}_{\boldsymbol{A}_{3}} \sqrt{2} \alpha_{2}+\underbrace{j\left[\begin{array}{cc}
0 & \frac{1}{2} \\
\frac{1}{2} & 0
\end{array}\right]}_{\boldsymbol{A}_{4}} \sqrt{2} \beta_{2},
\end{aligned}
$$

where $s_{1}=\alpha_{1}+j \beta_{1}$ and $s_{2}=\alpha_{2}+j \beta_{2}$ are two consecutive QPSK symbols per transmission block. As seen in Eq. (12), we may regard the QPSK-modulated Alamouti code as a BPSK-modulated G-STSK $(2,2,2,4,4)$ arrangement, employing $\boldsymbol{A}_{q^{\prime}}\left(q^{\prime}=1, \cdots, 4\right)$ of Eq. (12). By following a similar decomposition process, other OSTBCs may also be represented by our G-STSK system. Moreover, it may be readily shown that other STBCs, such as Quasi-OSTBCs (QSTBCs), STBC employing Time Variant Linear Transformation (TVLT) and Threaded Algebraic STBCs (TASTBCs), are also described by our G-STSK structure, according to Section 7.3 of [1].

4) BLAST: We may also view the BLAST architecture as a certain form of our G-STSK scheme, by setting $P=Q=M$, $T=1$ and using Eq. (7). This BLAST arrangement does not provide any explicit transmit diversity gain, and this property is shared by the SM/SSK schemes. Since the resultant system suffers from Inter-Antenna Interference (IAI) imposed on the AEs, the computational complexity of mitigating it becomes inevitably high, which increases with the number of AEs $M$.

5) STSK: Furthermore, in this contribution we refer to the special case of our G-STSK scheme, employing $P=1$, as STSK, where only one out of $Q$ dispersion matrices is activated, which results in lower $B_{1}$ and $B_{2}$ values in comparison to our G-STSK scheme for the case of $P>1$. This STSK arrangement enables us to implement single-streambased low-complexity ML detection, similarly to SM/SSK. Furthermore, an appropriately-constructed set of dispersion matrices $\boldsymbol{A}_{q^{\prime}}\left(q^{\prime}=1, \cdots, Q\right)$ enables us to dispense with symbol-level IAS. More specifically, the structure of each dispersion matrix $\boldsymbol{A}_{q^{\prime}}$ is constructed so that there is a single non-zero element for each column of the dispersion matrix $\boldsymbol{A}_{q^{\prime}}$. This constraint enables us to avoid any simultaneous transmission by multiple antennas, also similarly to SM/SSK.

\section{Detection Algorithm}

In this section, we present both the optimal hard-decision ML detector and the soft-detector, which are derived for our G-STSK system, assuming that perfect Channel State Information (CSI) is available at the receiver. The specific design of channel estimation schemes and the impact of channel estimation errors will be studies in our future work.

\section{A. Optimal Hard-Decision ML Detector}

Firstly, by applying the vectorial stacking operation vec( ) to the received signal block in Eq. (1), we arrive at

$$
\overline{\boldsymbol{Y}}(i)=\overline{\boldsymbol{H}}(i) \chi \boldsymbol{K}(i)+\overline{\boldsymbol{V}}(i),
$$

where we have $\overline{\boldsymbol{Y}}(i)=\operatorname{vec}[\boldsymbol{Y}(i)], \overline{\boldsymbol{H}}(i)=\boldsymbol{I} \otimes \boldsymbol{H}$, $\boldsymbol{\chi}=\left[\operatorname{vec}\left(\boldsymbol{A}_{1}\right), \cdots, \operatorname{vec}\left(\boldsymbol{A}_{Q}\right)\right]$ and $\overline{\boldsymbol{V}}(i)=\operatorname{vec}[\boldsymbol{V}(i)]$, while $\boldsymbol{I}$ is the identity matrix and $\otimes$ is the Kronecker product. Furthermore, the $q$ th element of the equivalent transmit vector $\boldsymbol{K}(i)$ is assumed to be $k_{q}(i)$. Here, if the $q$ th dispersion matrix $\boldsymbol{A}_{q}$ is selected in the $i$ th block as $\boldsymbol{A}^{(p)}(i)=\boldsymbol{A}_{q}, k_{q}(i)$ is set to the corresponding PSK/QAM symbol $s^{(p)}(i)$. Otherwise, $k_{q}(i)$ is zero. It should be noted that the number of non-zero components in $\boldsymbol{K}(i)=\left[k_{1}(i), \cdots, k_{Q}(i)\right]^{T}$ is equal to $P$.

Next, the conditional probability $P(\overline{\boldsymbol{Y}} \mid \boldsymbol{K})$ of the linearized equivalent system model of Eq. (13) is given by

$$
P(\overline{\boldsymbol{Y}} \mid \boldsymbol{K})=\frac{1}{\left(\pi N_{0}\right)^{N T}} \exp \left(-\frac{\|\overline{\boldsymbol{Y}}-\overline{\boldsymbol{H}} \boldsymbol{\chi} \boldsymbol{K}\|^{2}}{N_{0}}\right) .
$$

Accordingly, the ML detection criterion is formulated as

$$
\begin{aligned}
\left(\hat{B}_{1}, \hat{B}_{2}\right) & =\arg \max _{\left(B_{1}, B_{2}\right)} P(\overline{\boldsymbol{Y}} \mid \boldsymbol{K}) \\
& =\arg \min _{\left(B_{1}, B_{2}\right)}\|\overline{\boldsymbol{Y}}-\overline{\boldsymbol{H}} \boldsymbol{\chi} \boldsymbol{K}\|^{2} \\
& =\arg \min _{\left(B_{1}, B_{2}\right)}\left\|\overline{\boldsymbol{Y}}-\sum_{q=1}^{Q} k_{q}\{\overline{\boldsymbol{H}} \boldsymbol{\chi}\}_{q}\right\|^{2},
\end{aligned}
$$

where $\{\overline{\boldsymbol{H}} \boldsymbol{\chi}\}_{q}$ denotes the $q$ th column of $\overline{\boldsymbol{H}} \boldsymbol{\chi}$. Note that the computational complexity imposed by calculating $\sum_{q=1}^{Q} k_{q}\{\overline{\boldsymbol{H}} \boldsymbol{\chi}\}_{q}$ in Eq. (17) linearly increases with the parameter $P$, because the number of non-zero elements in $k_{q}(q=1, \cdots, Q)$ is $P$ as mentioned above.

More specifically, the computational complexity per bit for the detection scheme of Eq. (17) is evaluated in terms of the number of real-valued multiplications, which may be shown to be

$$
\frac{4 M N T^{2} Q+(4 N T P+2 N T) f(Q, P) \mathcal{L}^{P}}{B} .
$$

Fig. 4 shows the relationship between the complexity and the throughput of our QPSK-modulated G-STSK $(2,2,2,4, P)$ scheme designed for achieving the maximum diversity order 


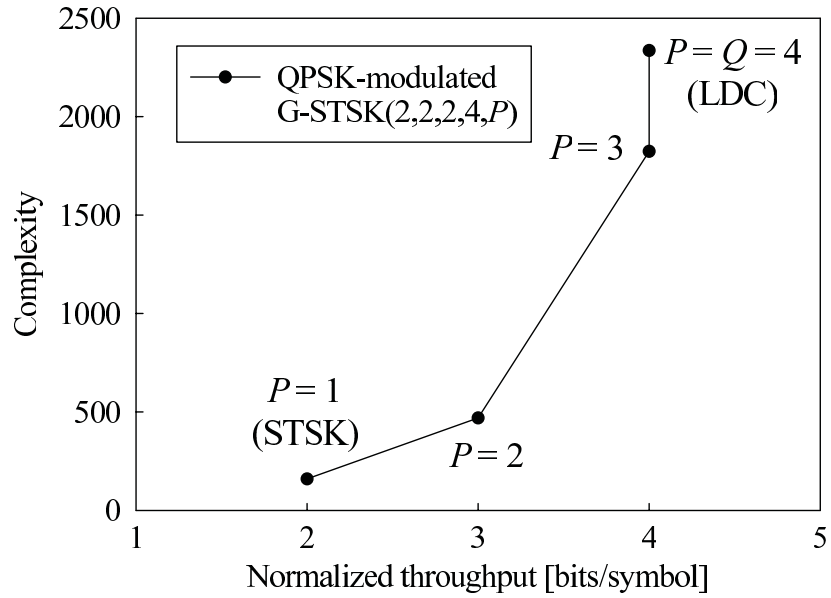

Fig. 4. The relationship between the complexity and the normalized throughput of our QPSK-modulated G-STSK $(2,2,2,4, P)$ scheme, which maintains a maximum diversity order of four. Here, the number of activated dispersion matrices $P$ was changed from $P=1$ to $P=4$.

of four, where the parameter $P$ was varied from $P=1$ to $P=4$. As mentioned in Section II, our G-STSK schemes employing $P=1$ and $P=Q$ correspond to the STSK and LDC schemes, respectively. As seen in Fig. 4, the normalized throughput $R$ tends to increase with the value of $P$ at the cost of an increased computational complexity.

\section{B. Soft Demodulator}

Although in Section III-A the optimal ML detector was derived for uncoded G-STSK systems, practical communication systems typically employ a powerful channel coding scheme, such as turbo coding [14]. Such a turbo-code assisted system is designed, where the iterative receiver exchanges soft extrinsic information between multiple decoders. Therefore, we hereby introduce the soft demodulator of our G-STSK scheme, which can be used for iterative detection assisted by Soft-Input Soft-Output (SISO) ${ }^{3}$ decoders. More specifically, the soft-demodulator processes the received signals and $a$ priori information is gleaned from the other decoder, in order to provide extrinsic information.

Considering that the equivalent received signal block $\overline{\boldsymbol{Y}}$ in Eq. (13) conveys channel-encoded binary bits $\boldsymbol{b}=$ $\left[b_{1}, b_{2}, \cdots, b_{B}\right]$, the resultant extrinsic Log-Likelihood Ratio (LLR) value $L_{\mathrm{e}}\left(b_{k}\right)$ of bit $b_{k}$ for $k=1,2, \cdots, B$ may be expressed as Eq. (20) [1] at the top of the following page, where $K_{1}^{k}$ and $K_{0}^{k}$ represent the sub-space of the legitimate equivalent signals $\boldsymbol{K}$, which satisfy $K_{1}^{k} \equiv\left\{\boldsymbol{K}_{\beta_{1}, \beta_{2}} \in K\right.$ : $\left.b_{k}=1\right\}$ and $K_{0}^{k} \equiv\left\{\boldsymbol{K}_{\beta_{1}, \beta_{2}} \in K: b_{k}=0\right\}$, respectively, while $\beta_{1} \in\left\{1, \cdots, 2^{B_{1}}\right\}$ and $\beta_{2} \in\left\{1, \cdots, 2^{B_{2}}\right\}$ are the variables corresponding to the $\mathrm{S} / \mathrm{P}$ converted $B_{1}$ and $B_{2}$ input bits of Fig. 2. Moreover, $L_{\mathrm{a}}\left(b_{j}\right)$ represents the a priori LLR value corresponding to the $j$ th bit.

Furthermore, Eq. (20) may be readily simplified by the wellknown max-log approximation [14], giving rise to Eq. (21) at the top of the following page.

\footnotetext{
${ }^{3}$ In the literature, the abbreviation of SISO may also be used for 'singleinput single-output' schemes, which are unrelated to the 'soft-input softoutput' solution used in this paper.
}

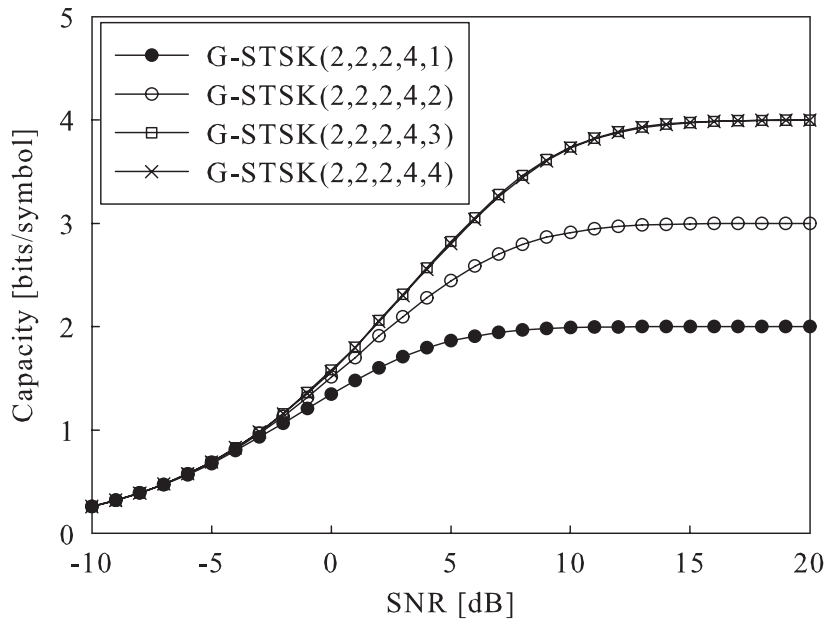

Fig. 5. DCMC capacity of the QPSK-modulated G-STSK $(2,2,2,4, P)$, where $P$ was varied from $P=1$ to $P=4$.

\section{DCMC CAPACITY}

In this section we characterize the DCMC capacity [10] of our G-STSK framework. As mentioned above, members of the G-STSK family support many other MIMO arrangements, hence the resultant capacity equation is also applicable to the class of MIMO arrangements shown in Fig. 1.

According to [10], the mutual information between input and output signals of our G-STSK scheme using $\mathcal{L}$-PSK/QAM signaling may be derived from that of the discrete memoryless channel as Eq. (22), which is shown on the next page. According to [15], under the assumption that all the signals $\boldsymbol{K}_{\beta_{1}, \beta_{2}}$ are equi-probable, i.e. when we have $P\left(\boldsymbol{K}_{1,1}\right)=\cdots=P\left(\boldsymbol{K}_{2^{B_{1}, 2^{B}}}\right)=1 / 2^{B}$, we arrive at Eq. (23), where we have

$$
\Psi_{\beta_{1}, \beta_{2}}^{\beta_{1}^{\prime}, \beta_{2}^{\prime}}=-\left\|\overline{\boldsymbol{H}} \chi\left(\boldsymbol{K}_{\beta_{1}, \beta_{2}}-\boldsymbol{K}_{\beta_{1}^{\prime}, \beta_{2}^{\prime}}\right)+\overline{\boldsymbol{V}}\right\|^{2}+\|\overline{\boldsymbol{V}}\|^{2} .
$$

By substituting Eq. (23) and $P\left(\boldsymbol{K}_{\beta_{1}, \beta_{2}}\right)=1 / 2^{B}$ into Eq. (22), we arrive at Eq. (24) [10], which is shown at the top of the page after the next page.

To be specific, in Fig. 5 we portray the DCMC capacity curves of our QPSK-modulated G-STSK $(2,2,2,4, P)$ scheme, where $P$ was varied from $P=1$ to $P=4$. As seen in Fig. 5, upon increasing the SNR value, each capacity curve converged at its attainable normalized throughput $R$ of Eq. (5). Additionally, observe in Fig. 5 that the capacity tended to be increased with the parameter $P$, although the capacity curves corresponding to $P=3$ and $P=4$ were found to be almost identical.

\section{Performance Results}

In this section the performance of our G-STSK system is characterized by conducting Monte Carlo simulations assuming a frequency-flat fast Rayleigh fading channel, while comparing the effects of a set of G-STSK parameters. In this contribution, we generated an appropriate dispersion-matrix set capable of achieving a good BER performance for each G-STSK arrangement, which were designed based on the well-known rank- and determinant-criterion [1] for the sake of simplicity, although we may readily employ other design 


$$
\begin{aligned}
L_{\mathrm{e}}\left(b_{k}\right)= & \ln \frac{\sum_{\boldsymbol{K}_{\beta_{1}, \beta_{2}} \in K_{1}^{k}} P\left(\overline{\boldsymbol{Y}} \mid \boldsymbol{K}_{\beta_{1}, \beta_{2}}\right) \cdot \exp \left[\sum_{j \neq k} b_{j} L_{\mathrm{a}}\left(b_{j}\right)\right]}{\sum_{\boldsymbol{K}_{\beta_{1}, \beta_{2}} \in K_{0}^{k}} P\left(\overline{\boldsymbol{Y}} \mid \boldsymbol{K}_{\beta_{1}, \beta_{2}}\right) \cdot \exp \left[\sum_{j \neq k} b_{j} L_{\mathrm{a}}\left(b_{j}\right)\right]} \\
= & \ln \frac{\sum_{\boldsymbol{K}_{\beta_{1}, \beta_{2}} \in K_{1}^{k}} \exp \left[-\left\|\overline{\boldsymbol{Y}}-\overline{\boldsymbol{H}} \boldsymbol{\chi} \boldsymbol{K}_{\beta_{1}, \beta_{2}}\right\|^{2} / N_{0}+\sum_{j \neq k} b_{j} L_{\mathrm{a}}\left(b_{j}\right)\right]}{\sum_{\boldsymbol{K}_{\beta_{1}, \beta_{2}} \in K_{0}^{k}} \exp \left[-\left\|\overline{\boldsymbol{Y}}-\overline{\boldsymbol{H}} \boldsymbol{\chi} \boldsymbol{K}_{\beta_{1}, \beta_{2}}\right\|^{2} / N_{0}+\sum_{j \neq k} b_{j} L_{\mathrm{a}}\left(b_{j}\right)\right]} .
\end{aligned}
$$

$$
\begin{aligned}
L_{\mathrm{e}}\left(b_{k}\right)= & \max _{\boldsymbol{K}_{\beta_{1}, \beta_{2}} \in K_{1}^{k}}\left[-\frac{\left\|\overline{\boldsymbol{Y}}-\overline{\boldsymbol{H}} \boldsymbol{\chi} \boldsymbol{K}_{\beta_{1}, \beta_{2}}\right\|^{2}}{N_{0}}+\sum_{j \neq k} b_{j} L_{\mathrm{a}}\left(b_{j}\right)\right] \\
& -\max _{\boldsymbol{K}_{\beta_{1}, \beta_{2}} \in K_{0}^{k}}\left[-\frac{\left\|\overline{\boldsymbol{Y}}-\overline{\boldsymbol{H}} \boldsymbol{\chi} \boldsymbol{K}_{\beta_{1}, \beta_{2}}\right\|^{2}}{N_{0}}+\sum_{j \neq k} b_{j} L_{\mathrm{a}}\left(b_{j}\right)\right] .
\end{aligned}
$$

$$
C=\frac{1}{T} \max _{P\left(\boldsymbol{K}_{1,1}, \cdots, P\left(\boldsymbol{K}_{2^{B_{1}, B_{2}}}\right)\right.} \sum_{\beta_{1}, \beta_{2}} \int_{-\infty}^{\infty} \cdots \int_{-\infty}^{\infty} P\left(\overline{\boldsymbol{Y}} \mid \boldsymbol{K}_{\beta_{1}, \beta_{2}}\right) P\left(\boldsymbol{K}_{\beta_{1}, \beta_{2}}\right) \log _{2}\left[\frac{P\left(\overline{\boldsymbol{Y}} \mid \boldsymbol{K}_{\beta_{1}, \beta_{2}}\right)}{\sum_{\beta_{1}^{\prime}, \beta_{2}^{\prime}} P\left(\overline{\boldsymbol{Y}} \mid \boldsymbol{K}_{\beta_{1}^{\prime}, \beta_{2}^{\prime}}\right) P\left(\boldsymbol{K}_{\beta_{1}^{\prime}, \beta_{2}^{\prime}}\right)}\right] d \overline{\boldsymbol{Y}}
$$

$$
\begin{aligned}
\log _{2}\left[\frac{P\left(\overline{\boldsymbol{Y}} \mid \boldsymbol{K}_{\beta_{1}, \beta_{2}}\right)}{\sum_{\beta_{1}^{\prime}, \beta_{2}^{\prime}} P\left(\overline{\boldsymbol{Y}} \mid \boldsymbol{K}_{\beta_{1}^{\prime}, \beta_{2}^{\prime}}\right) P\left(\boldsymbol{K}_{\beta_{1}^{\prime}, \beta_{2}^{\prime}}\right)}\right] & =-\log _{2}\left[\frac{1}{2^{B}} \sum_{\beta_{1}^{\prime}, \beta_{2}^{\prime}} \frac{P\left(\overline{\boldsymbol{Y}} \mid \boldsymbol{K}_{\beta_{1}^{\prime}, \beta_{2}^{\prime}}\right)}{P\left(\overline{\boldsymbol{Y}} \mid \boldsymbol{K}_{\beta_{1}, \beta_{2}}\right)}\right] \\
& =B-\log _{2}\left\{\sum_{\beta_{1}^{\prime}, \beta_{2}^{\prime}} \exp \left(\Psi_{\beta_{1}, \beta_{2}}^{\beta_{1}^{\prime}, \beta_{2}^{\prime}}\right)\right\} .
\end{aligned}
$$

criteria, such as the BLock Error Ratio (BLER) minimization technique of [16] and the DCMC-capacity maximization technique of [1]. More specifically, in order to obtain a desired dispersion-matrix set, we tentatively generated $10^{6}$ random dispersion-matrix sets, whose each element obeys the complex-valued Gaussian distribution. Then the best one out of the randomly-generated sets was selected according to the rank- and determinant-criterion. ${ }^{4}$

In Fig. 6 we firstly characterize the achievable BER performance of our uncoded and QPSK-modulated G$\operatorname{STSK}(2,2,2, Q, P)$ scheme, where the set of parameters $(Q, P)$ was given by $(Q, P)=(2,1),(4,1),(4,2),(4,3)$, $(4,4)$ as indicated by the legend. For comparison, we also included the QPSK-modulated G-STSK $(2,2,1,2,2)$ and the 8-PSK modulated G-STSK $(2,2,1,2,1)$ arrangements, which correspond to the classic BLAST and to the SM architectures , respectively. For completeness, we also added the theoretical upper bound derived based on Moment-Generating Functions (MGFs) [17] for each curve. Observe in Fig. 6 that the BER curves of our G-STSK systems exhibited the maximum

\footnotetext{
${ }^{4}$ Moreover, although we have employed the random search method for designing a good dispersion-matrix set, there are several approaches which may potentially implement its optimization in a more efficient manner. The detailed investigations are left for our future study.
}

diversity order of four, except for the cases of BLAST and SM. ${ }^{5}$ As expected, the corresponding BER performance was improved for both BLAST and SM upon reducing the normalized throughput from $R=4$ bits/symbol to $R=1.5$ bits/symbol.

Furthermore, in Figs. 7 and 8 we compared the GSTSK arrangements of Fig. 2 to other MIMOs, such as the STSK scheme of Section II-B5, the SM/SSK arrangement of Section II-B1, the orthogonal STBC of [18] and the BLAST scheme of Section II-B4. In Figs. 7 and $8,(M, N)=(3,2)$ AEs were employed, where the transmission rates were given by $R=2.0$ and 3.0 bits/symbol, respectively. ${ }^{6}$ Observe in Figs. 7 and 8 that our G-STSK scheme tended to outperform the STSK regime, which was the explicit benefit of its more flexible system design. However, the G-STSK's performance advantage over the STSK scheme was achieved at the expense of imposing on increased computational complexity invested in mitigating the effects of the $(P-1)$ ICI contributions. Furthermore, it was also confirmed in Figs. 7 and 8 that our G-STSK scheme had a higher BER performance, than

\footnotetext{
${ }^{5}$ Since BLAST and SM were not designed for achieving any transmit diversity gain, the performance advantage of other G-STSK $(2,2,2, Q, P)$ schemes over BLAST and SM increased upon increasing the SNR value.

${ }^{6}$ For reference, parts of the dispersion-matrix sets, which are used for the simulations, are shown in the Appendix.
} 


$$
\begin{aligned}
C & =\frac{B}{T 2^{B}} \sum_{\beta_{1}, \beta_{2}} \int_{-\infty}^{\infty} \cdots \int_{-\infty}^{\infty} P\left(\overline{\boldsymbol{Y}} \mid \boldsymbol{K}_{\beta_{1}, \beta_{2}}\right) d \overline{\boldsymbol{Y}}-\frac{1}{T 2^{B}} \sum_{\beta_{1}, \beta_{2}} \int_{-\infty}^{\infty} \cdots \int_{-\infty}^{\infty} P\left(\overline{\boldsymbol{Y}} \mid \boldsymbol{K}_{\beta_{1}, \beta_{2}}\right) \log _{2}\left\{\sum_{\beta_{1}^{\prime}, \beta_{2}^{\prime}} \exp \left(\Psi_{\beta_{1}, \beta_{2}}^{\beta_{1}^{\prime}, \beta_{2}^{\prime}}\right)\right\} d \overline{\boldsymbol{Y}} \\
& =\frac{1}{T}\left(B-\frac{1}{2^{B}} \sum_{\beta_{1}, \beta_{2}} E\left[\log _{2}\left\{\sum_{\beta_{1}^{\prime}, \beta_{2}^{\prime}} \exp \left(\Psi_{\beta_{1}, \beta_{2}}^{\beta_{1}^{\prime}, \beta_{2}^{\prime}}\right) \mid \boldsymbol{K}_{\beta_{1}^{\prime}, \beta_{2}^{\prime}}\right\}\right]\right) .
\end{aligned}
$$

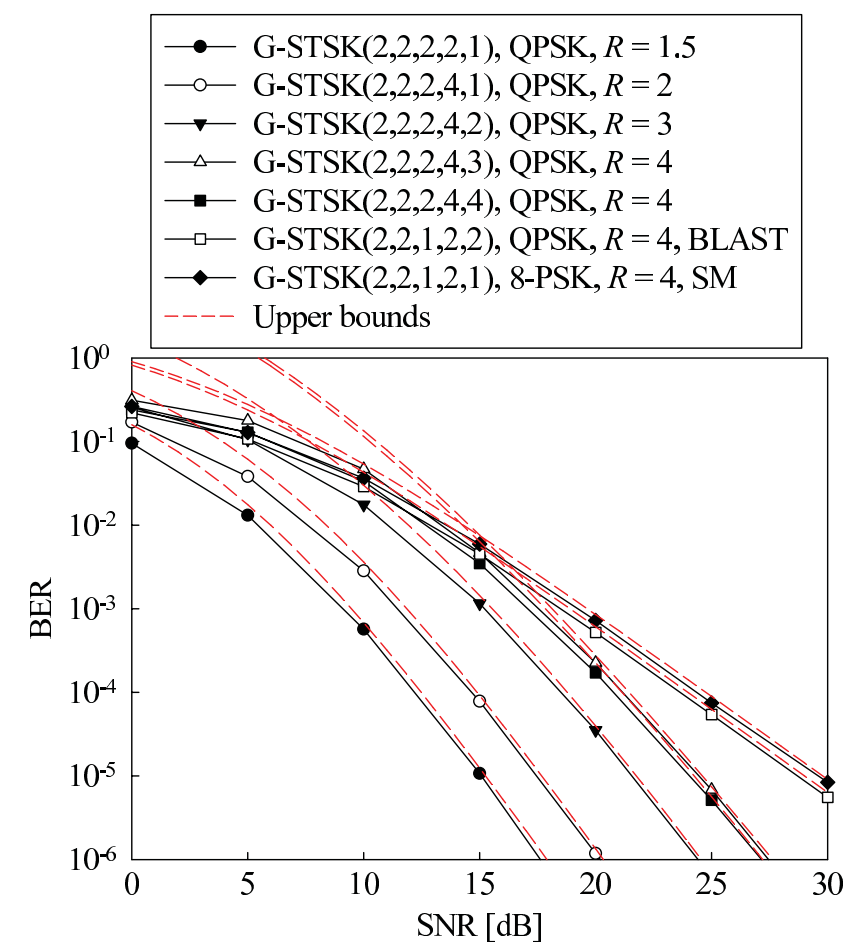

Fig. 6. Achievable BER performance of our uncoded QPSK-modulated G-STSK $(2,2,2, Q, P)$ scheme, where $(Q, P)$ were given by $(Q, P)=$ $(2,1),(4,1),(4,2),(4,3),(4,4)$.

conventional MIMO arrangements, such as the SM/SSK, the STBC and the BLAST schemes. We also note that although the STBC schemes achieved the maximum achievable diversity order of $M \cdot N$, the corresponding BER curves of Figs. 7 and 8 were inferior in comparison to the G-STSK and the STSK schemes. This is mainly due to the fact that the STBC schemes had to employ a high modulation order, in order to attain transmission rates that were comparable to those of the BLAST, GSTSK and STSK schemes.

Next, we investigated the coded G-STSK system as shown in Fig. 9, where we considered a serially concatenated threestage turbo codec characterized for example in Section 7.4 of [1]. More specifically, the information bits are firstly channelencoded by the half-rate Recursive Systematic Convolutional (RSC) code and then interleaved by the first random interleaver $\Pi_{1}$ of Fig. 9. Next, the interleaved bits are further encoded by the Unity-Rate Convolutional (URC) code and the URC-coded bits are then interleaved by another random interleaver $\Pi_{2}$ of Fig. 9. Finally, the interleaved bits are mapped to the AEs with the aid of our G-STSK mapping scheme of Fig. 2, in order to generate the space-time codewords $\boldsymbol{S}(i)$ to be transmitted to the receiver. By contrast, the receiver

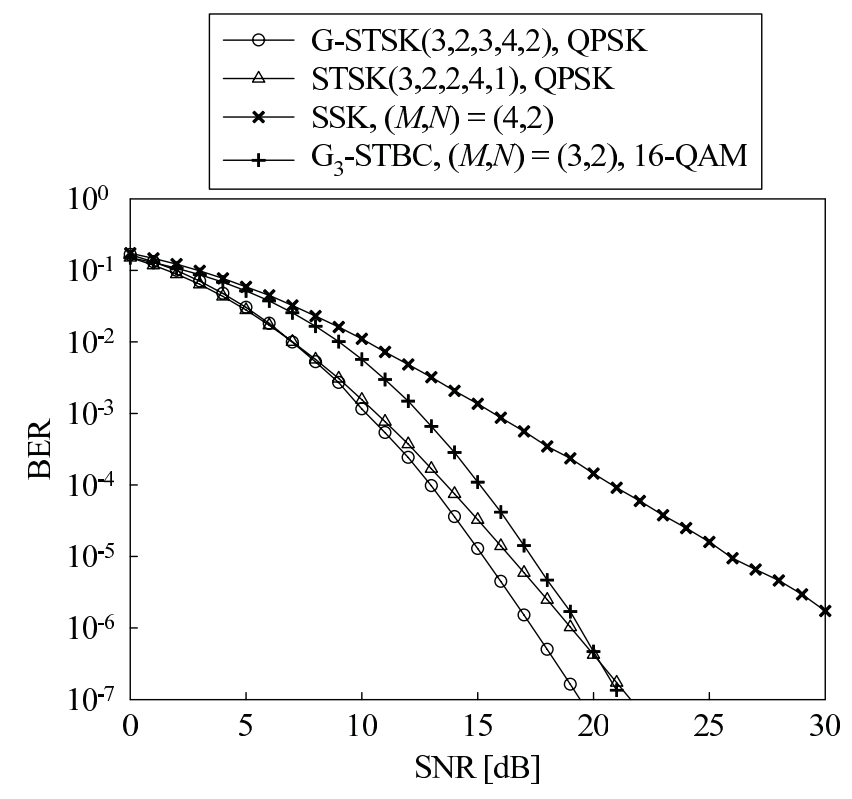

Fig. 7. BER comparison of our uncoded QPSK-modulated G$\operatorname{STSK}(3,2,3,4,2)$ scheme, the QPSK-modulated $\operatorname{STSK}(3,2,2,4,1)$ scheme, the SSK scheme [8] having $(M, N)=(4,2)$ antennas and the 16-QAM assisted $\mathrm{G}_{3}$-STBC scheme [18] having $(M, N)=(3,2)$ antennas. Here, each scheme exhibited a transmission rate of $R=2.0 \mathrm{bits} / \mathrm{symbol}$, while employing the optimal ML detection.

structure of Fig. 9 is constituted by a three-stage iterative detector, where three SISO decoders exchange their extrinsic information in the form of Log-Likelihood Ratios (LLRs). Let us assume that the RSC code is used as the outer code, while considering the amalgamated combination of the URC code and the G-STSK mapper to be the inner code.

Fig. 10 shows the EXIT curves of the QPSK-modulated G$\operatorname{STSK}(2,2,2, Q, P)$ arrangements at the SNR of $0 \mathrm{~dB}$, where the parameters $(Q, P)$ were varied. We also plotted the outer $\operatorname{RSC}(2,1,2)$ decoder's EXIT curve which employed the octal generator polynomials of $(3,2)_{8}$, and the EXIT trajectory associated with the $\operatorname{G-STSK}(2,2,2,3,2)$ scheme, where the interleaver length of both the interleavers $\Pi_{1}$ and $\Pi_{2}$ was set to 200000 bits. This is a high interleaver length, which enables a good match between the EXIT-chart prediction and the Monte-Carlo simulation-based BER results, as detailed in [1]. Furthermore, the corresponding EXIT curves recoded for BLAST and for the Alamouti code were also shown. It can be seen in Fig. 10 that depending on the G-STSK parameters employed, the corresponding inner decoder's EXIT curve exhibited substantially different characteristics. The area within the open EXIT-tunnel determines how close the system may operate with respect to the DCMC capacity. Particularly, 


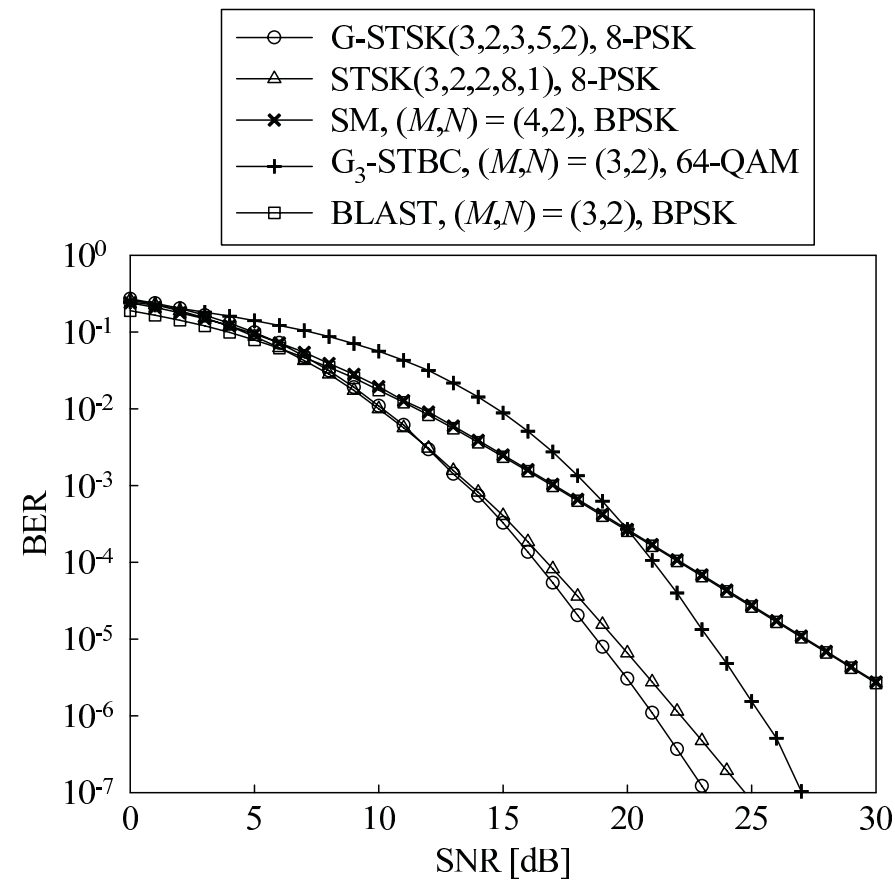

Fig. 8. BER comparison of our uncoded 8-PSK modulated G$\operatorname{STSK}(3,2,3,5,2)$ scheme, the 8-PSK modulated $\operatorname{STSK}(3,2,2,8,1)$, the BPSK-modulated SM scheme [9] having $(M, N)=(4,2)$ antennas, the 64QAM assisted $\mathrm{G}_{3}$-STBC scheme [18] having $(M, N)=(3,2)$ antennas and the BPSK-modulated BLAST scheme having $(M, N)=(3,2)$ antennas. Here, each scheme achieved 3.0 bits/symbol, while employing the optimal ML detection.

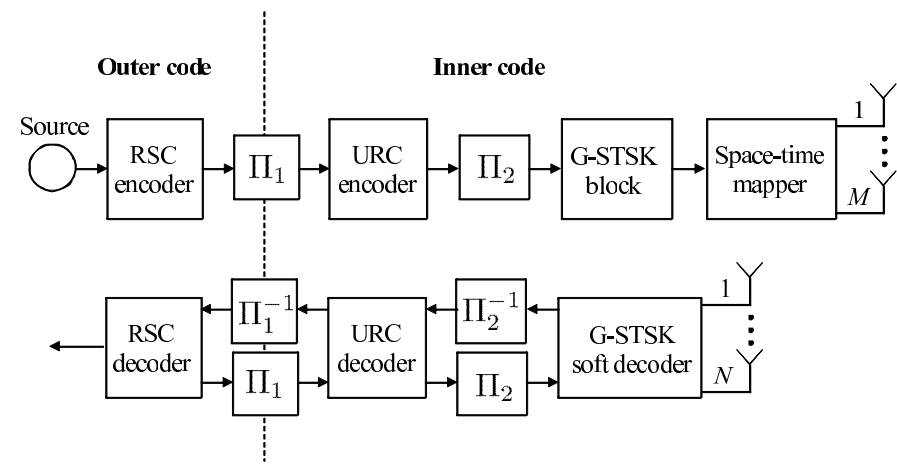

Fig. 9. Schematic of a three-stage RSC- and URC-coded G-STSK scheme using iterative detection.

the inner decoder's EXIT curves of the G-STSK $(2,2,2,3,2)$ scheme exhibited the narrowest open tunnel at this SNR point, resulting in a performance which was the nearest to capacity for all the G-STSK arrangements. Furthermore, the MonteCarlo simulation-based decoding trajectory demonstrated that the EXIT-chart prediction was quite accurate.

Finally, Fig. 11 shows the achievable BER performance of our RSC- and URC-coded G-STSK(2, 2, 2, 3,2) system employing QPSK modulation, achieving a total throughput of $R=1.25 \mathrm{bits} / \mathrm{symbol}$. The number of iterations $I$ between the outer and inner codes was varied from $I=0$ to $I=20$. As predicted from the EXIT chart of Fig. 10, the corresponding BER curve exhibited an infinitesimally low BER at the SNR point of $0 \mathrm{~dB}$. This was within about $1 \mathrm{~dB}$ from the SNR corresponding to the DCMC capacity, namely from $-1.0 \mathrm{~dB}$.

In order to attain an even 'nearer-to-capacity' performance,

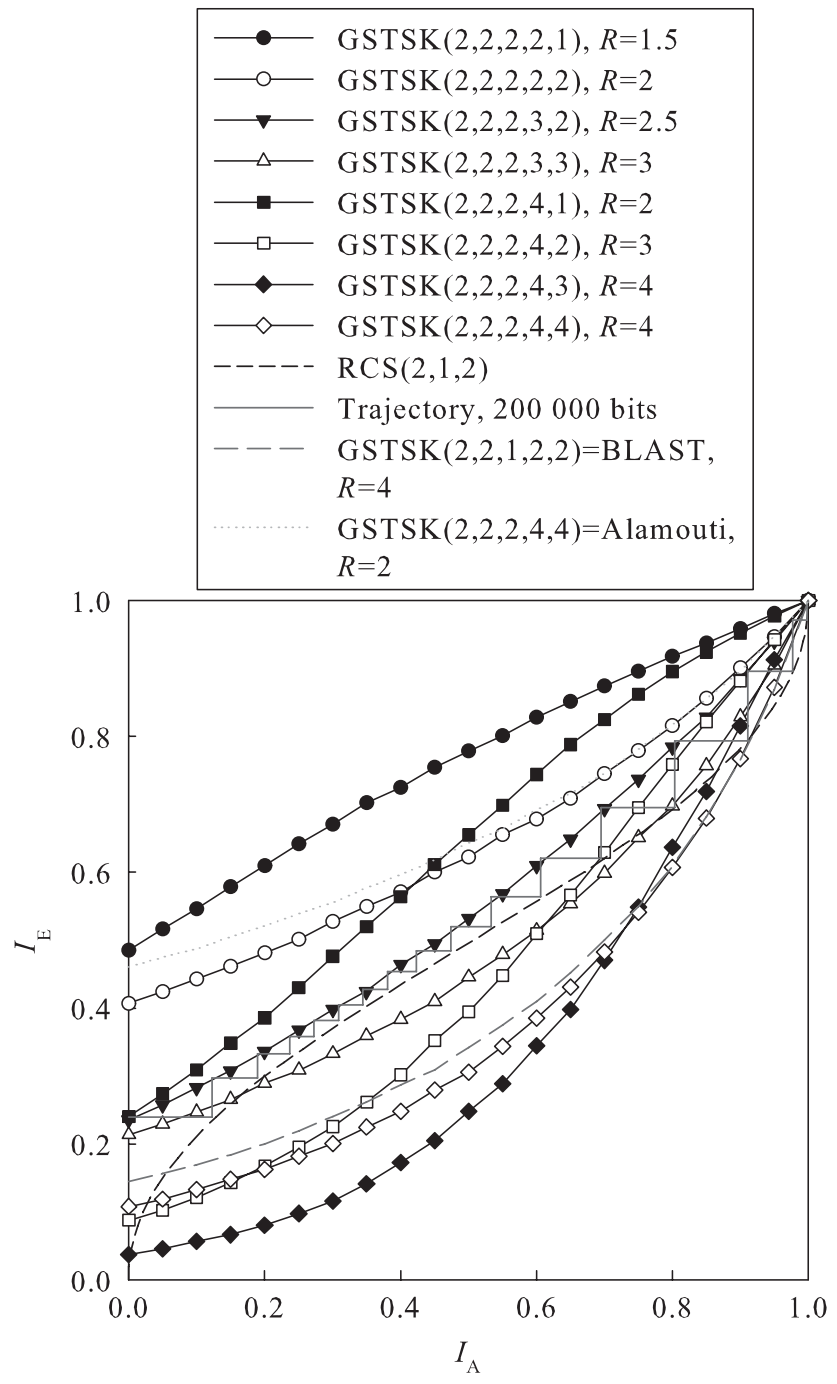

Fig. 10. EXIT chart of our RSC- and URC-coded G-STSK system.

the irregular inner- and outer-code concept [19] can be employed, where a set of EXIT curves corresponding to the diverse G-STSK parameters would allow us to create a narrower EXIT tunnel. However, it may be more practical to adaptively select one of the EXIT curves, in order to maintain an open EXIT tunnel, while increasing the achievable throughput $R$, depending on the instantaneous SNR. Additionally, we can also introduce a threshold for controlling the computational complexity imposed by the receiver, when appropriately designing the sets of G-STSK parameters configured for nearinstantaneously adaptive operation. This is particularly beneficial for a downlink scenario, where the mobile receiver's affordable processing capability is limited.

\section{CONCLUSIONS}

In this paper we proposed the novel G-STSK architecture, which acts as a unified MIMO framework, including many of the previously-developed MIMO arrangements, such as SM/SSK, LDC, STBC, BLAST and STSK as G-STSK's special cases. More specifically, based on the G-STSK's underlying concept, namely that $P$ out of $Q$ pre-allocated dispersion matrices are activated in conjunction with the $P$ 
This article has been accepted for inclusion in a future issue of this journal. Content is final as presented, with the exception of pagination.

$$
\begin{aligned}
& \boldsymbol{A}_{1}=\left[\begin{array}{rrr}
-0.1822+j 0.1836 & -0.2895-j 0.0459 & -0.3266+j 0.0084 \\
-0.5410-j 0.2791 & 0.1808+j 0.2297 & -0.2651-j 0.0169 \\
0.3534+j 0.1326 & -0.1305-j 0.4133 & -0.4618+j 0.4126
\end{array}\right], \\
& \boldsymbol{A}_{2}=\left[\begin{array}{rrr}
0.1842+j 0.1422 & -0.5593+j 0.2209 & -0.4014-j 0.1404 \\
0.4117-j 0.3931 & 0.0114+j 0.3390 & 0.1421+j 0.0166 \\
-0.4571-j 0.0340 & -0.1677+j 0.0778 & -0.1288-j 0.4278
\end{array}\right], \\
& \boldsymbol{A}_{3}=\left[\begin{array}{rrr}
-0.4247-j 0.0167 & -0.3032+j 0.5709 & 0.1076+j 0.0303 \\
0.2298-j 0.3942 & -0.1249-j 0.2413 & -0.0714-j 0.4140 \\
0.0966+j 0.3641 & -0.3253+j 0.1979 & 0.3606+j 0.1160
\end{array}\right], \\
& \boldsymbol{A}_{4}=\left[\begin{array}{rrr}
0.3281-j 0.1621 & 0.3511+j 0.0513 & -0.1194+j 0.1961 \\
0.0298-j 0.0971 & -0.1283-j 0.1433 & -0.8044-j 0.0415 \\
-0.0857-j 0.0824 & -0.4824+j 0.1671 & -0.3798-j 0.2691
\end{array}\right] .
\end{aligned}
$$

$$
\begin{aligned}
& \boldsymbol{A}_{1}=\left[\begin{array}{rrr}
0.3313-j 0.0934 & -0.2093+j 0.0059 & 0.3430-j 0.1426 \\
0.0256-j 0.2123 & 0.4258-j 0.1198 & -0.1492+j 0.3136 \\
-0.2001-j 0.1485 & -0.4746-j 0.7163 & 0.1930+j 0.0034
\end{array}\right], \\
& \boldsymbol{A}_{2}=\left[\begin{array}{rrr}
0.0533-j 0.5840 & -0.2676+j 0.0331 & 0.2379+j 0.2635 \\
-0.3727+j 0.1888 & -0.2517-j 0.3823 & 0.1967+j 0.1229 \\
-0.2409-j 0.0039 & 0.0660-j 0.0738 & -0.6645+j 0.1008
\end{array}\right], \\
& \boldsymbol{A}_{3}=\left[\begin{array}{rrr}
-0.4773+j 0.0533 & -0.1435+j 0.2393 & 0.5933-j 0.0776 \\
0.2637+j 0.0093 & -0.2280-j 0.4797 & 0.1906+j 0.0211 \\
0.2209-j 0.2538 & -0.0108-j 0.2367 & 0.5170-j 0.0911
\end{array}\right], \\
& \boldsymbol{A}_{4}=\left[\begin{array}{rrr}
0.1434+j 0.2098 & -0.3781-j 0.1050 & -0.5476+j 0.0826 \\
-0.0158+j 0.2100 & 0.1294-j 0.5712 & -0.3051+j 0.0995 \\
-0.1745+j 0.5746 & -0.1668-j 0.1640 & 0.0265-j 0.2613
\end{array}\right], \\
& \boldsymbol{A}_{5}=\left[\begin{array}{rrr}
0.1083+j 0.1886 & -0.3862+j 0.4963 & -0.3009+j 0.0908 \\
0.3435-j 0.4493 & -0.1621+j 0.0307 & 0.0856+j 0.0560 \\
0.0548-j 0.4980 & -0.2469+j 0.3589 & -0.3752-j 0.1402
\end{array}\right] .
\end{aligned}
$$

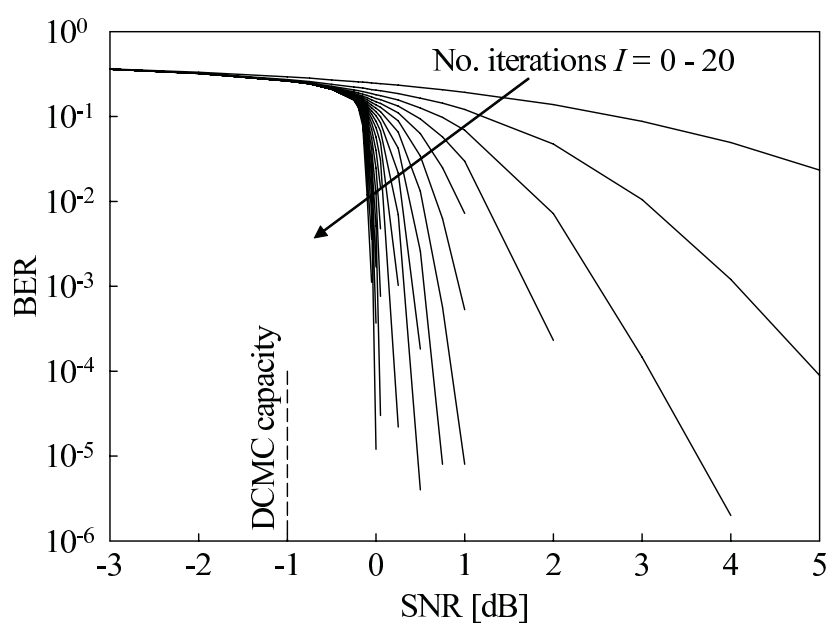

Fig. 11. Achievable BER performance of our RSC- and URC-coded G$\operatorname{STSK}(2,2,2,3,2)$ system employing QPSK modulation, where the number of iterations $I$ was changed from $I=0$ to $I=20$.

classic PSK/QAM symbols, we can strike a flexible tradeoff between the achievable diversity order, throughput as well as computational complexity. Additionally, the unified DCMC capacity was derived for our G-STSK scheme, which also represents the capacity of other MIMO arrangements. Our simulation results, including EXIT chart analysis and BER calculations, demonstrated that the proposed scheme was capable of near-capacity operation.

\section{APPENDIX \\ DisPersion-Matrix Set EMPloyed For OUR Simulations}

Parts of the dispersion-matrix sets $\boldsymbol{A}_{q^{\prime}}\left(q^{\prime}=1, \cdots, Q\right)$, which were used for our simulations in Figs. 7 and 8 are as follows. While the dispersion-matrix set of the QPSKmodulated G-STSK $(3,2,3,4,2)$ scheme are given by Eqs. (25)-(28), that of the 8-PSK modulated G-STSK $(3,2,3,5,2)$ scheme corresponds to Eqs. (29)-(33), which are shown at the top of this page.

\section{REFERENCES}

[1] L. Hanzo, O. Alamri, M. El-Hajjar, and N. Wu, Near-Capacity MultiFunctional MIMO Systems: Sphere-Packing, Iterative Detection and Cooperation. John Wiley and IEEE Press, 2009.

[2] P. Wolniansky, G. Foschini, G. Golden, and R. Valenzuela, "V-BLAST: an architecture for realizing very high data rates over the rich-scattering wireless channel," in Proc. International Symposium on Signals, Systems, and Electronics, 1998, pp. 295-300.

[3] V. Tarokh, N. Seshadri, and A. Calderbank, "Space-time codes for high data rate wireless communication: performance criterion and code construction," IEEE Trans. Inf. Theory, vol. 44, no. 2, pp. 744-765, 1998. 
[4] B. Hassibi and B. Hochwald, "High-rate codes that are linear in space and time," IEEE Trans. Inf. Theory, vol. 48, no. 7, pp. 1804-1824, 2002.

[5] R. Mesleh, H. Haas, C. Ahn, and S. Yun, "Spatial modulation-a new low complexity spectral efficiency enhancing technique," in Proc. First International Conference on Communications and Networking, Oct. 2006, pp. 1-5.

[6] R. Mesleh, H. Haas, S. Sinanovic, C. Ahn, and S. Yun, "Spatial modulation," IEEE Trans. Veh. Technol., vol. 57, no. 4, pp. 2228-2242, 2008.

[7] Y. A. Chau and S.-H. Yu, "Space modulation on wireless fading channels," in Proc. IEEE 54th Vehicular Technology Conference (VTC2001Fall), vol. 3, 2001, pp. 1668-1671.

[8] J. Jeganathan, A. Ghrayeb, L. Szczecinski, and A. Ceron, "Space shift keying modulation for MIMO channels," IEEE Trans. Wireless Commun., vol. 8, no. 7, pp. 3692-3703, 2009.

[9] J. Jeganathan, A. Ghrayeb, and L. Szczecinski, "Spatial modulation: optimal detection and performance analysis," IEEE Commun. Lett., vol. 12 , no. 8 , pp. 545-547, 2008.

[10] S. Ng and L. Hanzo, "On the MIMO channel capacity of multidimensional signal sets," IEEE Trans. Veh. Technol., vol. 55, no. 2, pp. 528536, 2006.

[11] S. ten Brink, "Convergence behavior of iteratively decoded paralle concatenated codes," IEEE Trans. Commun., vol. 49, no. 10, pp. 17271737, 2001.

[12] R. Heath Jr and A. Paulraj, "Linear dispersion codes for MIMO systems based on frame theory," IEEE Trans. Signal Process., vol. 50, no. 10, pp. 2429-2441, 2002.

[13] S. Alamouti, "A simple transmit diversity technique for wireless communications," IEEE J. Sel. Areas Commun., vol. 16, no. 8, pp. 1451-1458, 1998.

[14] L. Hanzo, T. Liew, and B. Yeap, Turbo Coding, Turbo Equalisation, and Space-Time Coding for Transmission over Fading Channels. John Wiley and IEEE Press, 2002.

[15] G. Caire, G. Taricco, and E. Biglieri, "Bit-interleaved coded modulation," IEEE Trans. Inf. Theory, vol. 44, no. 3, pp. 927-946, May 1998.

[16] J. Wang, X. Wang, and M. Madihian, "On the optimum design of spacetime linear-dispersion codes," IEEE Trans. Wireless Commun., vol. 4 no. 6, pp. 2928-2938, 2005.

[17] M. K. Simon and M. S. Alouini, Digital Communication over Fading Channels. Wiley-IEEE Press, 2005.

[18] V. Tarokh, H. Jafarkhani, and A. R. Calderbank, "Space-time block coding for wireless communications: performance results," IEEE J. Sel. Areas Commun., vol. 17, no. 3, pp. 451-460, 1999.

[19] N. Wu and L. Hanzo, "Near-capacity irregular-convolutional-codingaided irregular precoded linear dispersion codes," IEEE Trans. Veh. Technol., vol. 58, no. 6, pp. 2863-2871, 2009.

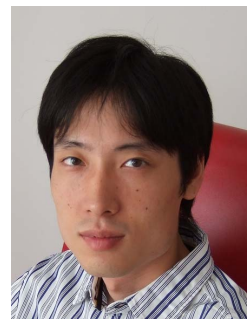

Shinya Sugiura (M'06) received the B.S. and M.S. degrees in aeronautics and astronautics from Kyoto University, Kyoto, Japan, in 2002 and 2004, respectively, and the Ph.D. degree in electronics and electrical engineering from University of Southampton, Southampton, UK, in 2010. In 2004, he joined Toyota Central Research and Development Laboratories (CRDL), Inc., Aichi, Japan. From 2007 to 2010, he was with the School of Electronics and Computer Science, University of Southampton, UK, on leave from Toyota CRDL, Inc. His research has covered a range of areas in communications, including space-time modulation and demodulation, turbo coding, cooperative communications, multiuser detection, automotive antenna design as well as vehicular ad hoc networking.

Dr. Sugiura has published over 25 research papers in various journals and conference proceedings. He was awarded IEEE AP-S Japan Chapter Young Engineer Award in December 2008

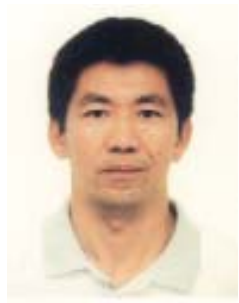

Sheng Chen (M'90-SM'97-F'08) obtained a BEng degree from the East China Petroleum Institute, Dongying, China, in 1982, and a $\mathrm{PhD}$ degree from the City University, London, in 1986, both in control engineering. In 2005, he was awarded DSc from the University of Southampton, Southampton, UK. Since 1999 he has been with the School of Electronics and Computer Science, the University of Southampton, UK. He previously held research and academic appointments at the Universities of Sheffield, Edinburgh and Portsmouth, all in UK. Professor Chen's recent research works include adaptive signal processing, wireless communications, modelling and identification of nonlinear systems, neural network and machine learning, finite-precision digital controller design, evolutionary computation methods, and optimization. He has published over 280 research papers. In the database of the world's most highly cited researchers in various disciplines, compiled by Institute for Scientific Information (ISI) of the USA, Prof. Chen is on the list of the highly cited researchers in the engineering category (see http://www.ISIHighlyCited.com),

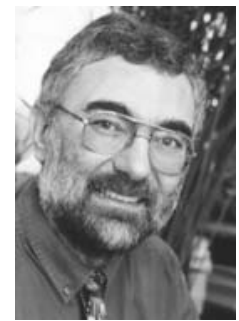

Lajos Hanzo (M'91-SM'92-F'04) FREng, FIEEE, FIET, DSc received his degree in electronics in 1976 and his doctorate in 1983. During his 35-year career in telecommunications he has held various research and academic posts in Hungary, Germany and the UK. Since 1986 he has been with the School of Electronics and Computer Science, University of Southampton, UK, where he holds the chair in telecommunications. He has co-authored 20 John Wiley - IEEE Press books on mobile radio communications totalling in excess of 10000 pages, published 969 research papers and book chapters at IEEE Xplore, acted as TPC Chair of IEEE conferences, presented keynote lectures and been awarded a number of distinctions. Currently he is directing an academic research team, working on a range of research projects in the field of wireless multimedia communications sponsored by industry, the Engineering and Physical Sciences Research Council (EPSRC) UK, the European IST Programme and the Mobile Virtual Centre of Excellence (VCE), UK. He is an enthusiastic supporter of industrial and academic liaison and he offers a range of industrial courses. $\mathrm{He}$ is also an IEEE Distinguished Lecturer as well as a Governor of the IEEE VTS. He is the Editor-in-Chief of the IEEE Press and a Chaired Prof. also at Tsinghua University, Beijing. For further information on research in progress and associated publications please refer to $\mathrm{http} / / / \mathrm{www}$-mobile.ecs.soton.ac.uk 